\title{
Overexpression of A disintegrin and metalloprotease 10 promotes tumor proliferation, migration and poor prognosis in hypopharyngeal squamous cell carcinoma
}

\author{
CHUANJIN DING ${ }^{*}$, QICHENG ZHANG ${ }^{*}$, YAN CHEN, XIAOBO ZHANG, PEI WU and ZHENXIN ZHANG \\ Department of Otorhinolaryngology Head and Neck Surgery, Affiliated Hospital of Nantong University, \\ Nantong, Jiangsu 226000, P.R. China
}

Received November 23, 2016; Accepted May 30, 2017

DOI: $10.3892 / o r .2017 .5761$

\begin{abstract}
The aim of this study was to determine the effect of A disintegrin and metalloprotease 10 (ADAM10) protein expression on the progression, migration and prognosis of hypopharyngeal squamous cell carcinoma (HSCC). Immunohistochemistry and western blot analysis were performed to detect ADAM10 expression in human HSCC specimens. Cell Counting Kit-8 (CCK-8) assay, flow cytometry analysis and wound-healing assay were employed to investigate the effects of ADAM10 knockdown (ADAM10-RNAi) on major oncogenic properties of FaDu cells. We detected that ADAM10 was overexpressed in HSCC specimens and its expression level was associated with differentiation $(\mathrm{p}<0.001)$, tumor size $(p=0.019)$, lymph node metastasis $(p=0.001)$, clinical stage $(\mathrm{p}<0.001)$, proliferation marker $\mathrm{Ki}-67$ expression $(\mathrm{P}=0.001)$ and overall survival $(\mathrm{p}<0.046)$. ADAM10-RNAi in $\mathrm{FaDu}$ cells resulted in the inhibition of proliferation and the decrease in migration. Moreover, mechanistic experiments revealed that ADAM10-RNAi resulted in an increase in E-cadherin and a decrease in N-cadherin and vimentin expression. Our study implies that high expression of ADAM10 promotes the proliferation and migration of HSCC. These findings may help to provide a method for treatment of HSCC.
\end{abstract}

\section{Introduction}

Hypopharyngeal squamous cell carcinoma (HSCC) is a common head and neck malignancy, accounting for 2-6\% of all head and neck cancers $(1,2)$. Due to the lack of evident

Correspondence to: Dr Zhenxin Zhang, Department of Otorhinolaryngology Head and Neck Surgery, Affiliated Hospital of Nantong University, Nantong, Jiangsu 226000, P.R. China

E-mail: dingchuanjin12@163.com

${ }^{*}$ Contributed equally

Key words: ADAM10, hypopharyngeal squamous cell carcinoma, progression, migration, prognosis clinical symptoms, easy lymph node metastasis and local infiltration in patients with early-stage $\operatorname{HSCC}(3,4)$, make treatment of HSCC one of the toughest challenges in human malignancies. Although the curative effect has progressively improved with the development of science and clinical technology in recent years, the overall survival has not improved due to the lymph node metastasis and distant metastasis of HSCC $(5,6)$. Therefore, studying the molecular mechanisms of HSCC progression and migration, would improve the diagnosis and prognosis of HSCC, and help to develop a new therapeutic strategy.

The A disintegrin and metalloprotease (ADAMs) family which has been reported in more than 30 species, and contains common structural features, consists of multidomain transmembrane proteases (7). ADAMs plays an important role in the degradation of intercellular adhesion, cell-matrix adhesion and basement membrane. Abberant expression of ADAMs is closely related to tumor proliferation, differentiation, adhesion, migration and invasion in tumors (7-11). ADAM10 is a crucial member of the ADAMs family $(12,13)$. The chief function of ADAM10 is shedding certain protein molecules in the extracellular region, thereby activating molecules, such as Ephrins (14), N-cadherin (15), E-cadherin (16), Notch receptor and its ligand $\delta 1$ (17). Previous research has revealed that ADAM10 is highly expressed in a variety of human types of cancer, including liver cancer (18), nasopharyngeal carcinoma (19), lung (20), gastric (21) and bladder cancer (22). Overexpression of ADAM10 promotes tumorigenesis and the progression, metastasis and invasion of tumors as well as the poor prognosis for cancer patients, by participating in a variety of signaling pathways (7-11,23-25). However, the expression of ADAM10 in HSCC and the role of ADAM10 on the progression, metastasis and poor prognosis of HSCC have yet to be elucidated.

In the present study, we first studied the expression of ADAM10 in HSCC, and the effect of ADAM10 expression on the proliferation and migration of HSCC. We found that ADAM10 overexpression in HSCC was associated with clinicopathological characteristics and the poor prognosis of patients with this disease. Moreover, inhibiting the expression level of ADAM10 decreased the proliferation and migration ability of the FaDu cell line. Our data indicated that ADAM10 
is a potential molecular target involved in the progression and metastasis mechanism of HSCC.

\section{Materials and methods}

Tissue specimens. All of the HSCC samples were collected from the Affiliated Hospital of Nantong University and pathologically diagnosed as HSCC by tissue biopsy. The clinical features of 46 patients with HSCC are listed in Table I. Fifteen pairs of fresh HSCC tissues and adjacent tissues were stored in $-80^{\circ} \mathrm{C}$ in a refrigerator. All selected patients had not undergone preoperative radiotherapy and chemotherapy. Informed consent was obtained from all individual participants included in the study. This study was approved by the Ethics Committee of the Affiliated Hospital of Nantong University and conformed to the provisions of the Declaration of Helsinki in 1995.

Immunohistochemical staining. Tissue samples were cut into paraffin sections for immunohistochemical staining. After deparaffinization and rehydration, the tissue sections were heated with $1 \mathrm{X}$ sodium citrate solution at $100^{\circ} \mathrm{C}$ for $30 \mathrm{~min}$ and washed 3 times. Then they were soaked in $3 \% \mathrm{H}_{2} \mathrm{O}_{2}$ for $15 \mathrm{~min}$ and subsequently washed 3 times. Finally the tissue sections were incubated with the primary antibodies overnight at $4^{\circ} \mathrm{C}$. A two-step incubation with a secondary antibody was performed using an immunohistochemistry universal kit (ZSGB-BIO, Beijing, China). DAB staining then followed and microscopic observation. Two pathologists assessed the staining intensity and percentage of stained cells in the tumor area, respectively. The expression level of ADAM10 was evaluated according to a semiquantitative scoring system named 'H-score approach' (26). The staining intensity was termed category A and assigned scores as strong (3); moderate (2); weak (1) or negative (0). The proportion of cells in the tumor area was termed category $\mathrm{B}$ and scored from 0 to 4: 0 (0\%); 1 (1-25\%); 2 (26-50\%); 3 (51-75\%) and 4 (76-100\%). A final score was calculated by multiplying A by $\mathrm{B}$ (minimum 0 , maximum 12). A final score of $<6$ was regarded as negative or weak expression, and a score of $\geq 6$ was regarded as high expression.

Cell lines, small interfering RNAs (siRNAs) and transfection. The FaDu cell line from our laboratory was cultured in Dulbecco's modified Eagle's medium (DMEM) supplemented with $10 \%$ fetal bovine serum (FBS) (both from Gibco, USA). Control non-targeted siRNAs and ADAM10-specific siRNAs were purchased from RiboBio (Guangzhou, China). Transfections were performed using Lipofectamine 2000 reagent (Invitrogen, USA) following the manufacturer's protocol.

Western blot analysis and antibodies. Protein was extracted from tissues and FaDu cells for protein analysis. The samples were boiled at $100^{\circ} \mathrm{C}$ for $5 \mathrm{~min}$. An equal amount of protein samples was separated by SDS-PAGE and transferred to PVDF membranes (Millipore, USA). The membranes were incubated with primary antibodies overnight at $4^{\circ} \mathrm{C}$. The antibodies used were as follows: anti-ADAM10 (1:300, polyclonal, rabbit anti-human; BBI Life Sciences Corporation, Shanghai, China), anti-Ki-67 (1:1,000; polyclonal, rabbit anti-human), anti-E-cadherin $(1: 1,000)$, anti-N-cadherin (1:1,000, monoclonal, mouse anti-human), anti-vimentin
Table I. Clinicopathological characteristics of HSCC patients and IHC staining score for ADAM10.

\begin{tabular}{|c|c|c|c|}
\hline Groups & $\mathrm{N}$ & $\begin{array}{c}\text { ADAM10 IHC } \\
\text { score }^{\mathrm{a}}\end{array}$ & P-value ${ }^{b}$ \\
\hline \multicolumn{4}{|l|}{ Age (years) } \\
\hline$<60$ & 20 & $5.80 \pm 3.736$ & 0.741 \\
\hline$\geq 60$ & 26 & $6.15 \pm 3.461$ & \\
\hline \multicolumn{4}{|l|}{ Sex } \\
\hline Male & 43 & $6.05 \pm 3.605$ & 0.740 \\
\hline Female & 3 & $5.33 \pm 3.055$ & \\
\hline \multicolumn{4}{|l|}{ Differentiation } \\
\hline Keratinizing & 20 & $3.90 \pm 2.278$ & $<0.001^{\mathrm{c}}$ \\
\hline Non-keratinizing & 26 & $7.62 \pm 3.238$ & \\
\hline \multicolumn{4}{|l|}{ Tumor size (cm) } \\
\hline$\leq 2$ & 15 & $4.27 \pm 3.390$ & $0.019^{c}$ \\
\hline$>2$ & 31 & $6.84 \pm 3.358$ & \\
\hline \multicolumn{4}{|l|}{ Clinical stage } \\
\hline I-II & 18 & $3.67 \pm 2.701$ & $<0.001^{\mathrm{c}}$ \\
\hline III-IV & 28 & $7.50 \pm 3.226$ & \\
\hline \multicolumn{4}{|c|}{ Lymph node metastasis } \\
\hline Negative & 19 & $3.95 \pm 2.896$ & $0.001^{\mathrm{c}}$ \\
\hline Positive & 27 & $7.44 \pm 3.274$ & \\
\hline \multicolumn{4}{|l|}{ Ki-67 expression } \\
\hline Low & 31 & $4.45 \pm 3.064$ & $0.001^{\mathrm{c}}$ \\
\hline High & 15 & $9.20 \pm 2.007$ & \\
\hline
\end{tabular}

${ }^{\mathrm{a}} \mathrm{ADAM} 10 \mathrm{IHC}$ score expressed as the mean \pm SD. ${ }^{\mathrm{b}}$ Student's t-test for two groups. 'Statistically significant, $\mathrm{P}<0.05$. ADAM10, A disintegrin and metalloprotease 10 ; HSCC, hypopharyngeal squamous cell carcinoma.

(1:1,000, monoclonal, mouse anti-human), and anti- $\beta$-actin polyclonal antibody (1:2,500, monoclonal, mouse anti-human) (all from Santa Cruz Biotechnology, USA). Immunoreactive protein bands were detected using an ECL detection system (Cell Signaling Technology, USA).

Cell proliferation assay. The CCK-8 assay was used to detect cell proliferation. The cells were seeded in 96-well plates $(10,000$ cells/well), incubated overnight, and then transfected with siRNA-ADAM10 or control non-targeted siRNAs and cultured for 12, 24, 36, 48, 60 and $72 \mathrm{~h}$. The CCK-8 kit reagent ( $10 \mu \mathrm{l} /$ well; BBI Life Sciences Corporation) was added and incubation followed in the dark in an incubator for $2 \mathrm{~h}$. Subsequently the OD was assessed at $450 \mathrm{~nm}$.

Cell cycle analysis. Flow cytometry was used to analyze the effect of ADAM10 on cell cycle progression. Briefly, $\mathrm{FaDu}$ cells were cultured in 6-well plates. The medium was replaced with $10 \% \mathrm{FBS}$ at $6,12,24$ and $36 \mathrm{~h}$ and after serum starvation at $72 \mathrm{~h}$, or siRNA-ADAM10 interference for $48 \mathrm{~h}$. The adherent cells were collected by trypsin digestion and centrifuged at $95 \mathrm{x} \mathrm{g}$ for $5 \mathrm{~min}$. The cells were then washed twice with phosphate-buffered saline (PBS), and fixed with 

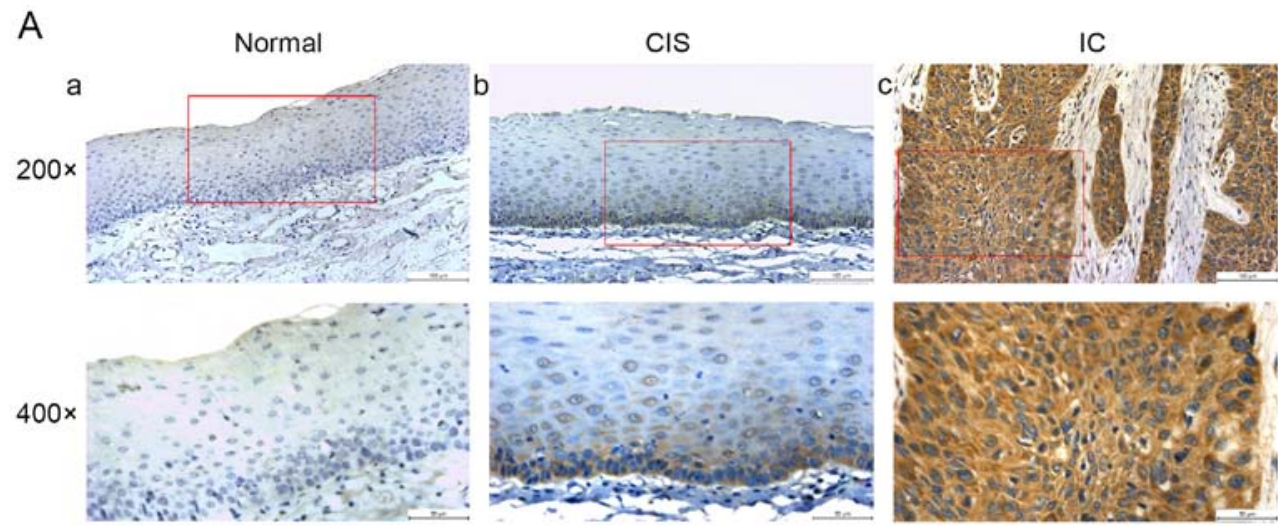

$\mathrm{B}$

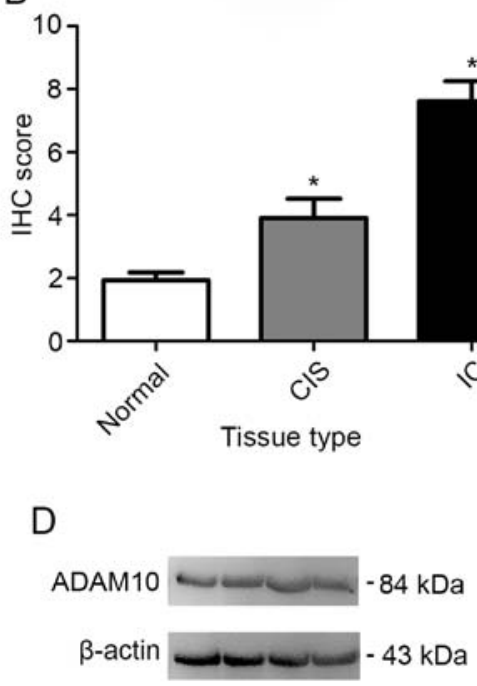

C

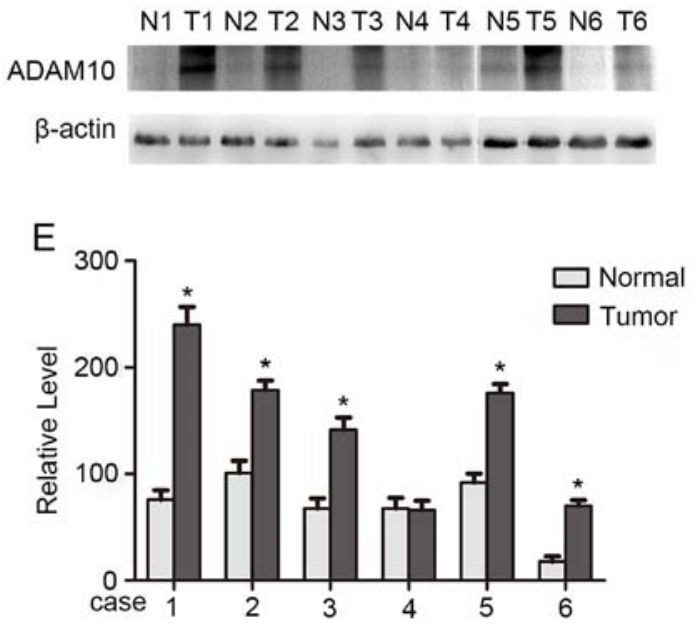

Figure 1. Expression level of ADAM10 in paracancerous and HSCC tissues. (A) Representative images of ADAM10 in paracancerous and HSCC tissues. a) Low ADAM10 expression in paracancerous tissue; b) moderate ADAM10 expression in CIS; and c) high ADAM10 expression in IC (magnification x200, scale bars, $100 \mu \mathrm{m}$; magnification x 400, scale bars, $50 \mu \mathrm{m}$ ). (B) The IHC score of ADAM10 expression in samples displayed in A. The mean \pm SD was used to express the data. ${ }^{*}<0.05$. (C) The protein levels of ADAM10 in 6 out of 15 paired HSCC (T) and non-cancerous hypopharyngeal tissues (N) by western blotting. (D) ADAM10 protein expression was detected abundantly in FaDu cells by western blot analysis. (E) Quantitative results of the western blot analysis in C. ${ }^{*} \mathrm{P}<0.05$. ADAM10, A disintegrin and metalloprotease 10; HSCC, hypopharyngeal squamous cell carcinoma.

$70 \%$ alcohol. The cells were then stored at $-20^{\circ} \mathrm{C}$ for $24 \mathrm{~h}$, washed with PBS 2 times and resuspended in $1 \mathrm{ml}$ of $1 \mathrm{X}$ PBS containing $0.1 \%$ Triton X-100, $40 \mu \mathrm{g} / \mathrm{ml}$ RNase and $20 \mu \mathrm{g} / \mathrm{ml}$ propidium iodide (PI) followed by a 30 -min incubation at room temperature. Finally, the samples were analyzed using the FACSCalibur flow cytometer (BD Biosciences, USA) and BD CellQuest software.

Migration assay. A wound healing assay was performed for cell migration. The cells were seeded in 6-well plates and incubated overnight, then transfected with siRNA-ADAM10 or control non-targeted siRNAs and a scratch wound was made using a $200-\mu 1$ yellow tip when the cells reached $\sim 90 \%$ density. Subsequently the cells were cultured with serum-free DMEM medium for $36 \mathrm{~h}$. The percentage of migration was calculated based on the measured cell-free area. Similarly, Transwell migration assays were performed for cell migration. FaDu cells were transfected with siRNA-ADAM10 or control non-targeted siRNAs and seeded to the upper chamber containing serum-free DMEM with a non-coated membrane (24-well insert, $8-\mu \mathrm{m}$ pore size; Millipore) and DMEM containing $10 \%$ serum was added to the lower chamber. After $24 \mathrm{~h}$, the cells from the upper chamber were removed and the cells that had migrated to the lower chamber were fixed with formaldehyde, and then stained with $0.1 \%$ crystal violet. The cells were then counted using an IX70 inverted microscope.

Statistical analysis. Statistical software (IBM SPSS Statistics 20; IBM SPSS, Armonk, NY, USA) was used for statistical analysis. The Student's t-test was used to determine the statistical differences between groups. $\mathrm{P}<0.05$ was considered statistically significant.

\section{Results}

ADAM10 protein overexpression in HSCC specimens. It was reported that ADAM10 is aberrant in tumors (18-22). Here, we first detected the expression of ADAM10 in HSCC. Immunohistochemical analysis of ADAM10 expression in hypopharyngeal normal tissues and tumor tissues revealed that ADAM10 was weakly expressed in normal tissues, moderately expressed in cancer in situ (CIS) and highly 
A

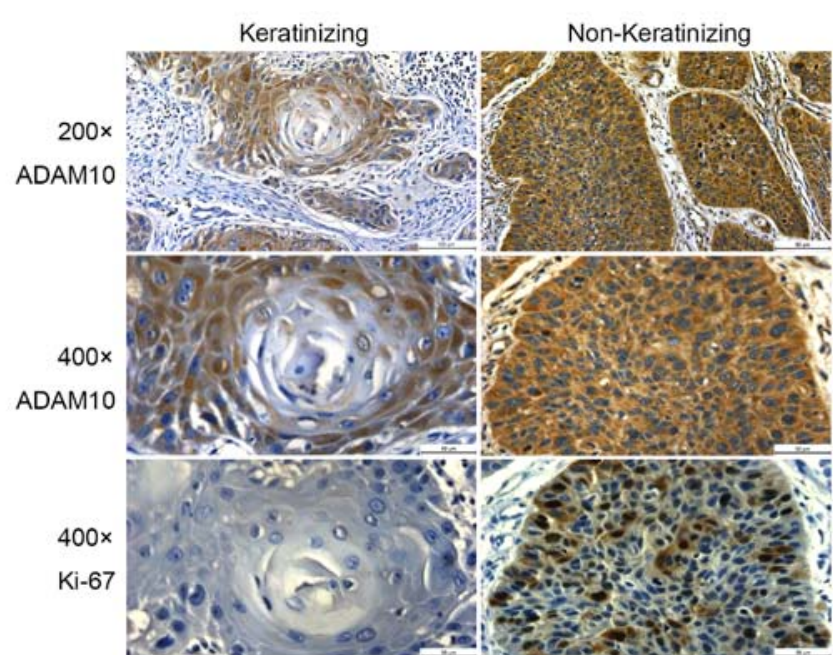

B

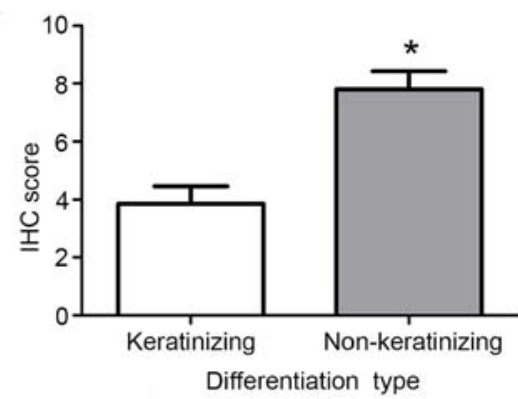

D

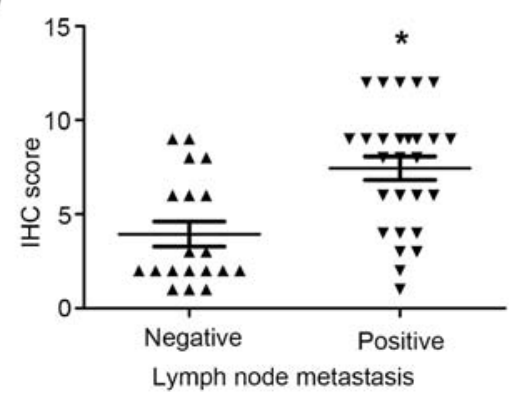

C

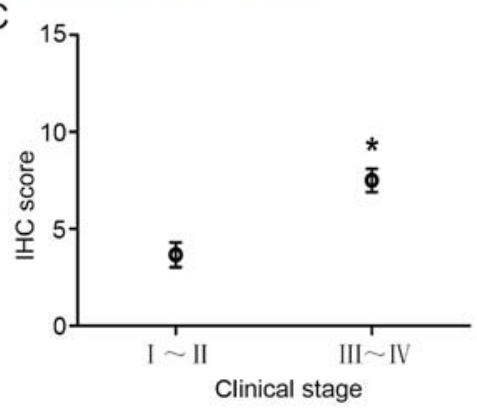

E

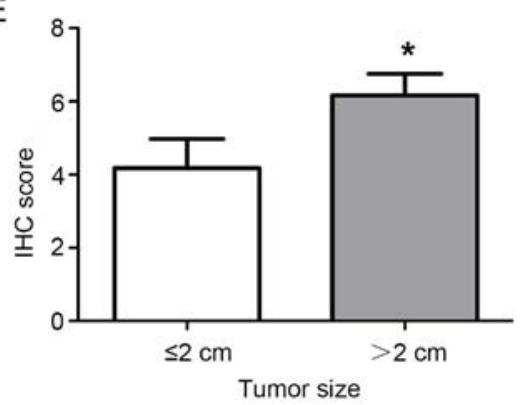

Figure 2. Relationship between ADAM10 expression and clinicopathological characteristics. (A) Representative images of ADAM10 IHC staining score in different differentiation types, and the relationship between the Ki-67 proliferation index and the ADAM10 expression level (magnification x 200 , scale bars, $100 \mu \mathrm{m}$; magnification $\mathrm{x} 400$, scale bars, $50 \mu \mathrm{m}$ ). (B) The ADAM10 IHC score was significantly higher in the non-keratinizing type than that in the keratinizing type as shown in A. Moreover, the ADAM10 IHC score was significantly correlated with (C) clinical stages, (D) lymph node metastasis and (E) tumor size. The mean $\pm \mathrm{SD}$ was used to express the data. ${ }^{*} \mathrm{P}<0.05$. ADAM10, A disintegrin and metalloprotease 10.

expressed in invasive cancer (IC) (Fig. 1A). The IHC score of ADAM10 was significantly decreased in normal tissues $(1.93 \pm 0.88)$ compared with that in CIS $(3.90 \pm 2.79, \mathrm{P}<0.05)$ or IC $(7.62 \pm 3.24, \mathrm{P}<0.05)$ (Fig. 1B). In addition, there was a statistical difference in the IHC score of ADAM10 between CIS and IC tissues $(\mathrm{P}<0.05)$. Subsequently, we examined the expression of ADAM10 in 15 pairs of HSCC and precancerous tissues by western blot analysis, which revealed that the expression level of ADAM10 in cancer tissues was significantly higher than that in adjacent tissues (Fig. 1C and E). Finally, we also detected the expression of ADAM10 in the HSCC cell line FaDu (Fig. 1D). The results revealed that ADAM10 was overexpressed and may promote tumorigenesis and progression in HSCC.

Correlation of ADAM10 expression level with clinicopathological features. We further assessed the correlation of the expression level of ADAM10 with clinicopathological characteristics in HSCC. As shown in Table I and Fig. 2, high expression of ADAM10 in HSCC was significantly correlated with the degree of tumor differentiation $(\mathrm{p}<0.001)$. Squamous cell carcinoma is divided into keratinizing and non-keratinizing squamous cell carcinoma (27). In addition, overexpression of ADAM10 in HSCC was also associated with tumor size $(p=0.019)$, lymph node metastasis $(p=0.001)$ and clinical stage $(p<0.001)$. There was no correlation with the sex and age of the patients and HSCC. Moreover, we also analyzed the correlation of the expression level of ADAM10 with Ki-67 expression $(\mathrm{p}=0.001)$. The result revealed that there was a significant positive correlation in the expression level of ADAM10 with the expression of Ki-67 (Fig. 3A). The data revealed that the expression of ADAM10 had a significant correlation with the clinicopathological characteristics of HSCC. Thus, high expression of ADAM10 may promote proliferation and migration. 
A

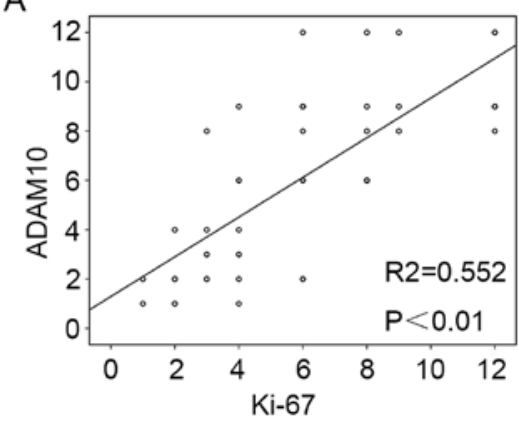

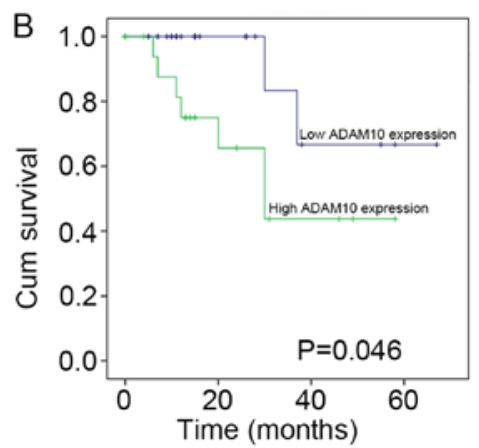

Figure 3. Scatter plots and Kaplan-Meier overall survival curve. Scatter plots/ regression lines in (A) demonstrates a linear correlation for Ki-67 proliferation index and ADAM10 expression in HSCC (Spearman's correlation coefficient P<0.01). (B) Kaplan-Meier overall survival curves for patients with HSCC for low (blue) and high (green) protein expression of ADAM10. Patients in the high expression group had a significantly poorer prognosis than those in the low expression group ( $\mathrm{P}=0.046$, log-rank test). ADAM10, A disintegrin and metalloprotease 10; HSCC, hypopharyngeal squamous cell carcinoma.

A
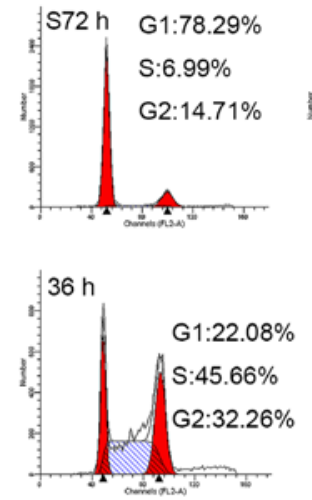

C

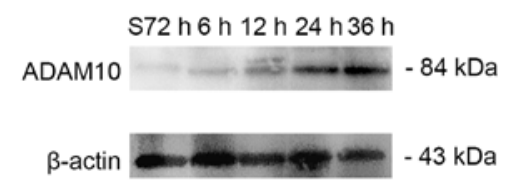

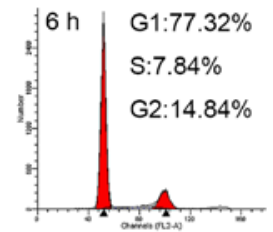
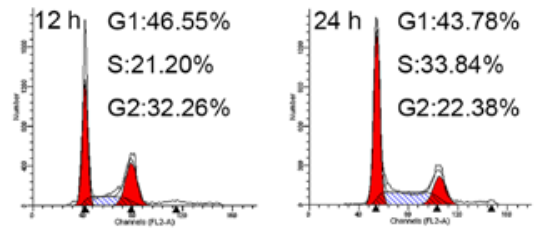

$\mathrm{B}$

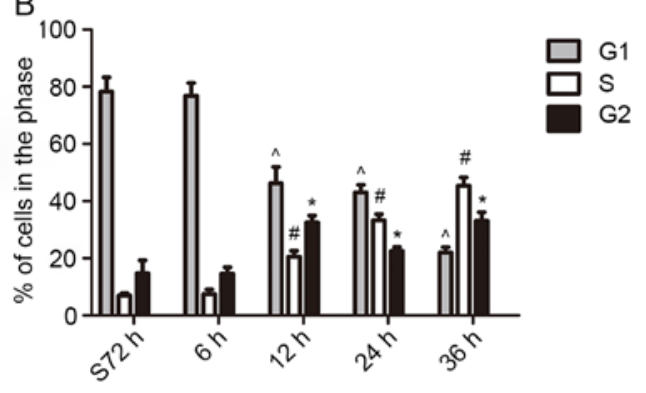

D

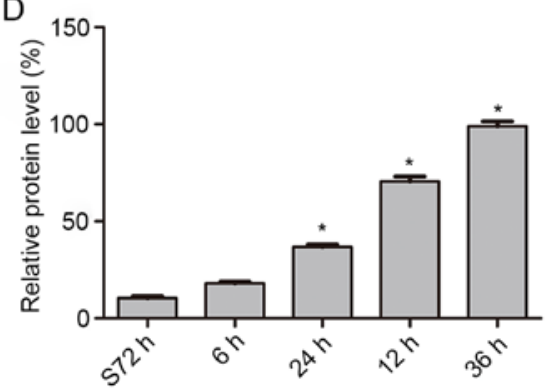

Figure 4. Expression level of ADAM10 in proliferating HSCC FaDu cells. (A) Serum was released into the medium, after cells were serum-starved for $72 \mathrm{~h}$. Flow cytometry was used to detect the percentage of cells in the different phases of the cell cycle. (B) Quantitative results of the percentage of cells in the different phases shown in A. (C) Serum was released into medium, after cells were serum-starved for $72 \mathrm{~h}$. The expression of ADAM10 was analyzed by western blot analysis after cell lysates were collected. (D) Quantitative results of the western blot analysis as shown in C. $\beta$-actin was used as a loading control The mean \pm SD was used to express the data. ${ }^{* \wedge} \mathrm{P}<0.05$ vs. S72 h. ADAM10, A disintegrin and metalloprotease 10; HSCC, hypopharyngeal squamous cell carcinoma.

High expression of ADAM10 is associated with the poor prognosis of HSCC patients. Similarly, we analyzed the relationship between the expression of ADAM10 and the prognosis of 46 patients with HSCC by statistical analyses. The result revealed that overall survival was significantly decreased in patients with high expression of ADAM10 than in those with low expression of ADAM10 (Fig. 3B) $(\mathrm{p}<0.046)$. It is therefore implied that overexpression of ADAM10 led to the poor prognosis of patients with HSCC.
Expression of ADAM10 in proliferating HSCC cells. In order to further explore the possible biological roles of ADAM10 in HSCC we examined the expression level of ADAM10 in proliferating FaDu cells. First, we cultured FaDu cells in serumfree medium for $72 \mathrm{~h}$ to stop the cell cycle in the G1 phase. Then, we collected the cells after being cultured with $10 \%$ FBS at 0 , $6,12,24$, and $36 \mathrm{~h}$. Flow cytometric analysis revealed that the percentage of cells in the $S$ phase of the cell cycle increased from 6.99 to $45.66 \%$ (Fig. 4A and B). Similarly, the expression level 
A

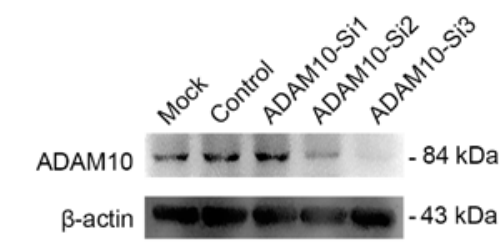

$\mathrm{C}$

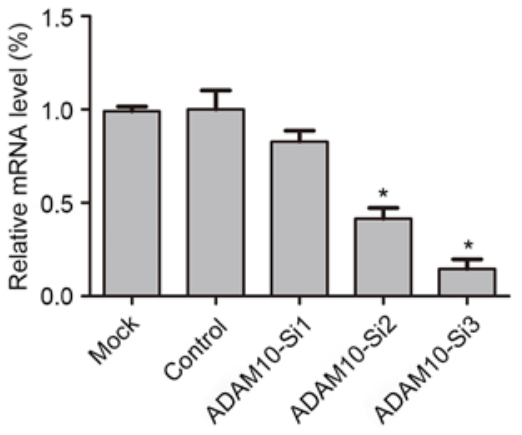

B

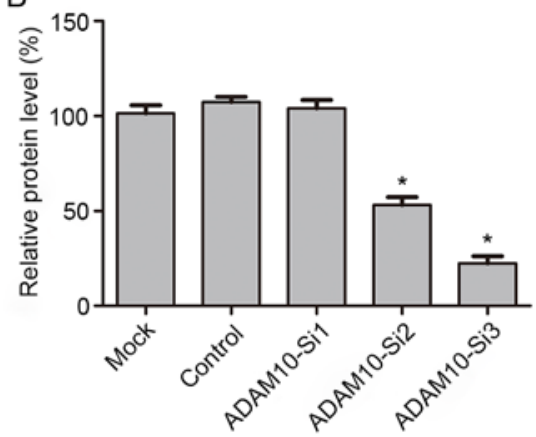

D

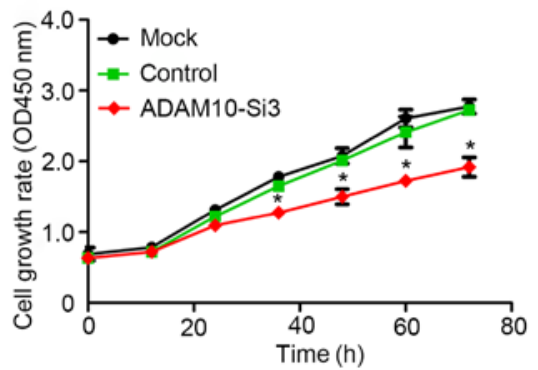

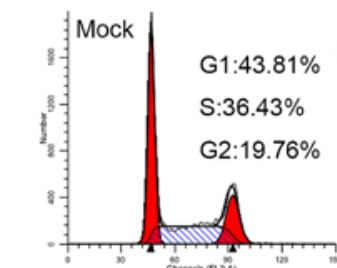
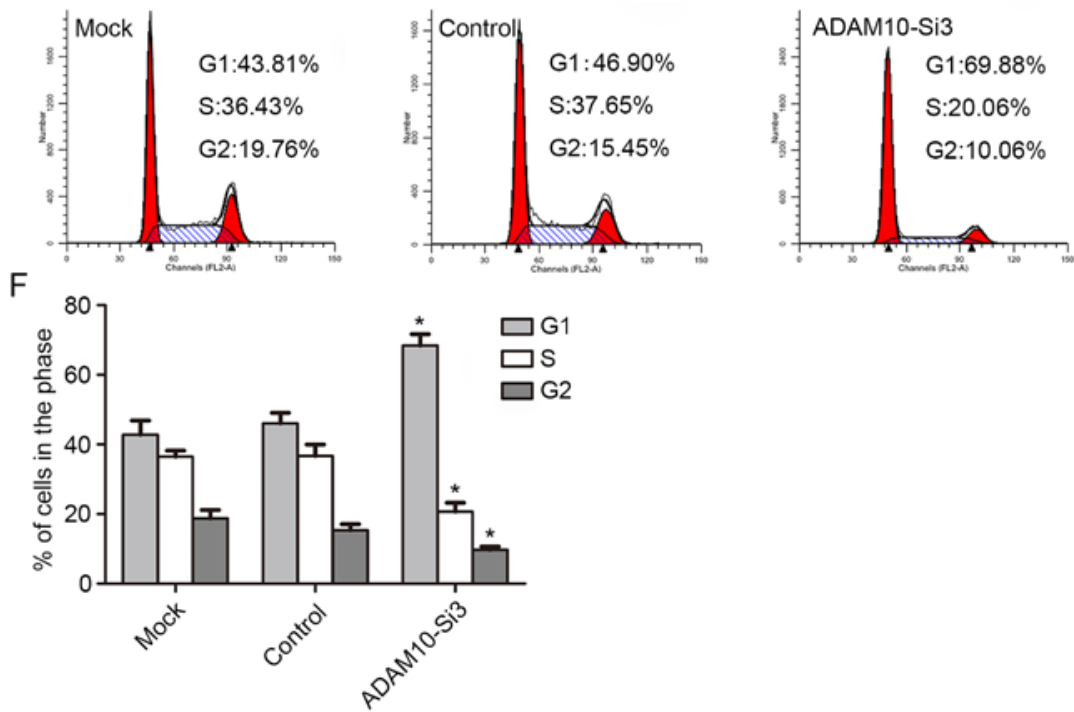

Figure 5. Effects of ADAM10-Si3 on the proliferation of FaDu cells. (A-C) Western blot analysis and real-time PCR were used to detect the effect of ADAM10 knockdown. (D) CCK-8 assay was used to assess cell proliferation. (E and F) Flow cytometry was used to analyze the cell cycle in FaDu cells. Data are expressed as the mean \pm SEM. "P<0.05. ADAM10, A disintegrin and metalloprotease 10; CCK-8, Cell Counting Kit-8; ADAM10-Si3, ADAM10-RNA3.

of ADAM10 in the cells was obviously increased as detected by western blot analysis (Fig. 4C and D). The result revealed that ADAM10 was involved in the process of cell proliferation.

The proliferation of FaDu cells was attenuated by knockdown of ADAM10. The above studies revealed that ADAM10 could promote the proliferation of HSCC. Therefore, FaDu cells were transfected with ADAM10 siRNAs to decrease the expression of ADAM10 in order to detect the function of ADAM10 in HSCC. First, we assessed the knockdown efficiency by western blotting and real-time PCR. The results revealed that the expression level of ADAM10 in the FaDu cell line was significantly decreased after knockdown, especially in ADAM10-siRNA3 (Fig. 5A-C). Subsequently, we examined cell proliferation by CCK- 8 assay. The result revealed that the cell proliferation ability was significantly decreased after knockdown with ADAM10-siRNA3 (Fig. 5D). The cell cycle was investigated by flow cytometric analysis. The results revealed that the percentage of cells in the G0/G1 phase increased significantly and the percentage of cells in the $\mathrm{S}$ phase decreased significantly after knockdown of ADAM10 (Fig. 5E and F). Thus, it was concluded that ADAM10 can promote the growth of HSCC.

The migration of FaDu cells was inhibited by knockdown of $A D A M 10$. We then examined the migration ability of FaDu cells with ADAM10-siRNA3 knockdown by wound-healing and Transwell assay. The study revealed that wound healing was significantly decreased by ADAM10-siRNA3 compared with the control group after $36 \mathrm{~h}$ (Fig. 6A and B). Similarly, Transwell assay revealed that the number of migratory cells were significantly lower in the ADAM10-siRNA3 group than 
A

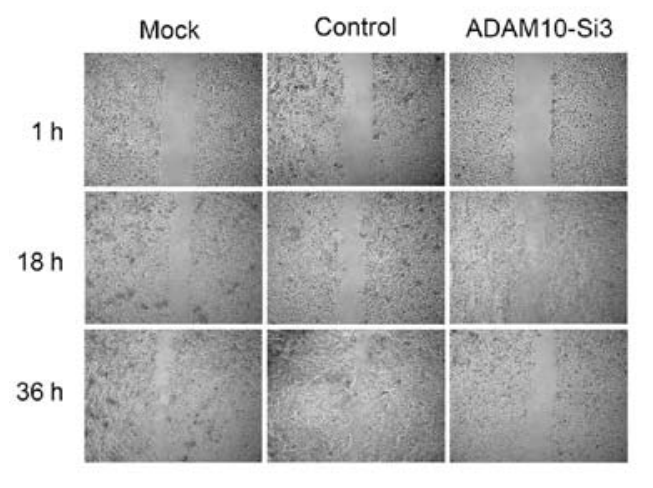

B

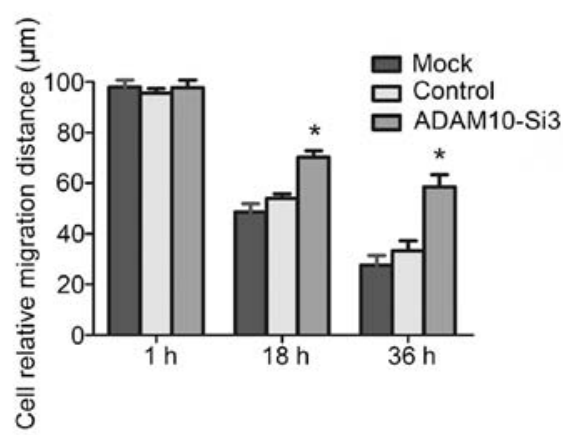

C

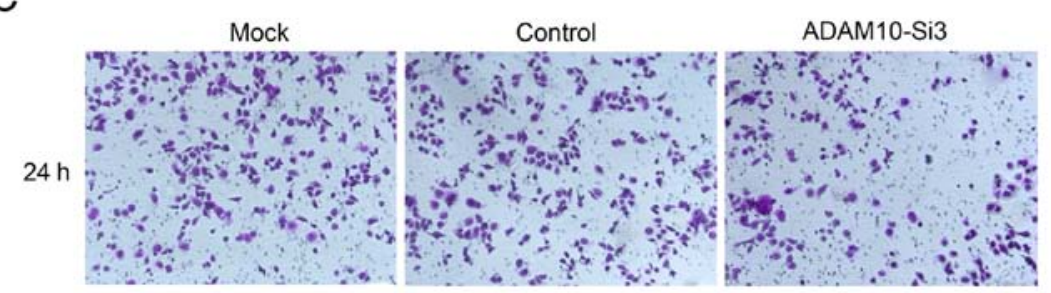

D

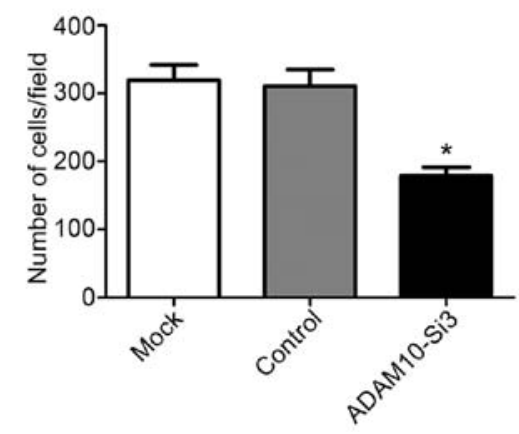

E

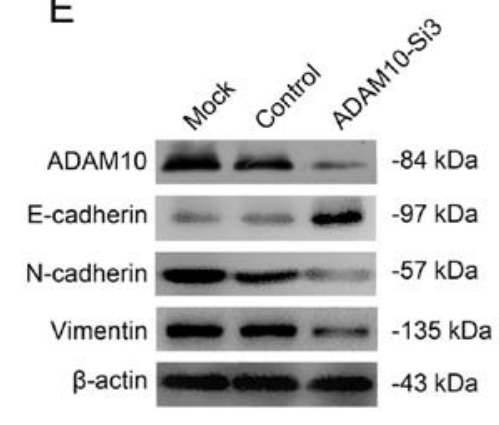

Figure 6. Effects of ADAM10-Si3 on the migration of FaDu cells. Migration of FaDu cells was detected by wound-healing assay and Transwell assay after transfection with ADAM10-Si3 or non-specific siRNA or control. (A and B) Decreased healing was observed by wound-healing assay after transfection with ADAM10-Si3. (C and D) Fewer cells passed through the microporous membrane as determined by Transwell assay after transfection with ADAM10-Si3. (E) The expression level of E-cadherin, N-cadherin, and vimentin was detected by western blot analysis in FaDu cells after transfection with ADAM10-Si3. Data are expressed as the mean \pm SEM. ${ }^{*} \mathrm{P}<0.05$. ADAM10, A disintegrin and metalloprotease 10; ADAM10-Si3, ADAM10-RNA3.

those in the control group (Fig. 6C and D). In conclusion, the migration ability of FaDu cells was significantly decreased after knockdown of ADAM10.

In the process of tumor migration, epithelial-mesenchymal transition (EMT) plays a decisive role (28). It could decrease cell adhesion, eliminate cell polarity, empower cell motility and invasive ability, thus leading to the metastasis of tumor cells from the original tumor location to adjacent organs and distant organs (29). The EMT process is usually accompanied with decreased expression of epithelial markers (E-cadherin, cytokeratin, and $\beta$-catenin) and increased expression of mesenchymal phenotypic markers (vimentin, $\mathrm{N}$-cadherin and fibronectin), which play important roles in cell adhesion $(29,30)$. Therefore, we detected the expression of $\mathrm{N}$-cadherin, vimentin and E-cadherin in $\mathrm{FaDu}$ cells after knockdown with ADAM10-siRNA3. The result revealed that the expression of E-cadherin was increased and N-cadherin and vimentin were decreased in the FaDu cells after treatment with ADAM10-siRNA3 (Fig. 6E). The result demonstrated that ADAM10 promoted tumor migration by affecting EMT.

\section{Discussion}

In our present study, we investigated whether ADAM10 had a potential effect on HSCC. To clarify the effect of ADAM10 on HSCC, we performed immunohistochemical and western blot analysis to detect the expression of ADAM10 and its relationship with clinicopathological characteristics. In addition, we observed the effect of the expression of ADAM10 on the proliferation and migration ability of the FaDu cell line. Collectively, our results revealed that ADAM10 was highly expressed and promoted tumor progression, migration and the poor prognosis of patients with HSCC.

ADAM10, a member of the ADAMs family, is a multinomial transmembrane protease, which is widely expressed in the body (31-34). The main function of ADAM10 is ectodomain shedding on a variety of transmembrane receptors, cytokines and adhesion molecules, such as TNF- $\alpha$, EGF, E-cadherins, N-cadherins and Notch (15,16,31-35). High expression of ADAM10 has been reported in a variety of malignancies and is involved in the development and migration 
of tumors (7-11,16,23-25). However, the role of ADAM10 in HSCC has not been reported yet.

In this study, we found that ADAM10 was highly expressed in CIS and IC of HSCC compared with normal tissues, likewise, the expression level of IC was also higher than that of CIS (Fig. 1). Moreover, we found that the expression level of ADAM10 was correlated with tumor size, differentiation, clinical stage and lymph node metastasis in HSCC (Fig. 2). It is suggested that ADAM10 may be a potential oncogene and play an important role in the tumorigenesis and progression of HSCC. The aberrant expression of ADAM10 may be involved in tumor cell proliferation and migration-related signaling pathways, thus promoting tumor growth, lymph node metastasis and distant metastasis. These results were similar with those of You et al (19) that reported the effect of ADAM10 in nasopharyngeal carcinoma and Liu et al (36) that reported the effect of ADAM10 in hepatocellular carcinoma.

The studies revealed that the prognosis of HSCC was mainly related to the clinical stage, malignancy and lymph node metastasis and distant metastasis $(5,6)$. Thus, we analyzed the effect of ADAM10 on the prognosis of HSCC. The results revealed that overexpression of ADAM10 significantly decreased the time of survival. Overall survival was significantly decreased in HSCC patients with high expression of ADAM10 compared to those with low expression (Fig. 3B). Combined with the above results (Figs. 1 and 2) it is implied that high expression of ADAM10 may promote migration and invasion, increase the difficulty of treatment and enhance the recurrence rate in $\mathrm{HSCC}$, thus decreasing the overall survival rate of HSCC patients.

To further test our hypothesis, we used the HSCC cell line FaDu for validation. First, we detected the expression level of ADAM10 in proliferating FaDu cells, which revealed that the percentage of cells in the $S$ phase increased with the increase of ADAM10 expression (Fig. 4). Subsequently, we knocked down the expression of ADAM10 in FaDu cells, The results revealed that the proliferation ability of FaDu cells (Fig. 5D) and the percentage of $\mathrm{S}$ phase cells (Fig. 5E and F) were significantly decreased after knockdown of ADAM10 expression. It was further demonstrated that the expression of ADAM10 promoted the abnormal proliferation of cells in HSCC. Furthermore, we investigated the effect of ADAM10 on the migration of FaDu cells. It was revealed that the ability of scratches to heal (Fig. 6A and B) and the number of cells that passed through the membrane (Fig. 6C and D) were markedly decreased after knockdown of ADAM10 expression in $\mathrm{FaDu}$ cells. These results indicated that downregulation of ADAM10 expression can significantly inhibit the movement and migration of FaDu cells. In other words, high expression of ADAM10 may promote the proliferation and migration of tumors in HSCC.

Tumor invasion and metastasis are very complex molecular regulation processess. The current study determined that EMT plays a decisive role in these processes (28). EMT is mainly characterized by the decrease of cell epithelial characteristics, the increase of interstitial characteristics, remodeling of the cytoskeleton and disappearance of cell matrix adhesion; and then a decrease of cell adhesion, elimination of cell polarity, empowerement of cell motility and invasive ability (29). This process is usually accompanied by decreased expression of epithelial phenotypic markers (e.g., E-cadherin and $\beta$-catenin) and increased expression of interstitial phenotypic markers (e.g., vimentin, and N-cadherin) (29,30). We employed proteomic analysis and found that the expression of E-cadherin was significantly increased, and the expression of vimentin and N-cadherin were significantly decreased after downregulation of ADAM10 expression in FaDu cells (Fig. 6E). The results suggested that high expression of ADAM10 may increase EMT regulation, and promote tumor cell migration and infiltration in HSCC.

In conclusion, complex biological processes are involved in the unrestricted tumor growth and metastasis of HSCC. Therefore, it is very important to understand the biological mechanism of the proliferation and metastasis of HSCC, which could detect the potential genes that promote the proliferation and metastasis of HSCC. In this study, our data clearly revealed that ADAM10 was highly expressed in HSCC and promoted the proliferation, migration and the poor prognosis of patients with HSCC. Thus, ADAM10 may be an important molecular target of HSCC proliferation and metastasis, with potentially important therapeutic implications.

\section{Acknowledgements}

We would like to thank Zhifeng Gu for the experimental help, and the Jiangsu Province Key Laboratory of Neuroregeneration of Nantong University for their support. This study was supported by the People's Livelihood Scientific and Technological Innovation and Demonstration Promotion, Nantong, Jiangsu (MS32016015).

\section{References}

1. Ligier K, Belot A, Launoy G, Velten M, Bossard N, Iwaz J, Righini CA, Delafosse P and Guizard AV; network Francim: Descriptive epidemiology of upper aerodigestive tract cancers in France: Incidence over 1980-2005 and projection to 2010. Oral Oncol 47: 302-307, 2011.

2. Takes RP, Strojan P, Silver CE, Bradley PJ Jr, Haigentz M Jr, Wolf GT, Shaha AR, Hartl DM, Olofsson J, Langendijk JA, et al; International Head and Neck Scientific Group: Current trends in initial management of hypopharyngeal cancer: The declining use of open surgery. Head Neck 34: 270-281, 2012.

3. Hoffman HT, Karnell LH, Shah JP, Ariyan S, Brown GS, Fee WE, Glass AG, Goepfert H, Ossoff RH and Fremgen AM: Hypopharyngeal cancer patient care evaluation. Laryngoscope 107: 1005-1017, 1997.

4. Godballe C, Jørgensen K, Hansen O and Bastholt L: Hypopharyngeal cancer: Results of treatment based on radiation therapy and salvage surgery. Laryngoscope 112: 834-838, 2002.

5. Lee MS, Ho HC, Hsiao SH, Hwang JH, Lee CC and Hung SK: Treatment results and prognostic factors in locally advanced hypopharyngeal cancer. Acta Otolaryngol 128: 103-109, 2008.

6. Chung EJ, Lee SH, Baek SH, Park IS, Cho SJ and Rho YS: Pattern of cervical lymph node metastasis in medial wall pyriform sinus carcinoma. Laryngoscope 124: 882-887, 2014.

7. Seals DF and Courtneidge SA: The ADAMs family of metalloproteases: Multidomain proteins with multiple functions. Genes Dev 17: 7-30, 2003.

8. Mochizuki S and Okada Y: ADAMs in cancer cell proliferation and progression. Cancer Sci 98: 621-628, 2007.

9. Lu X, Lu D, Scully M and Kakkar V: ADAM proteins - therapeutic potential in cancer. Curr Cancer Drug Targets 8: 720-732, 2008.

10. Murphy G: The ADAMs: Signalling scissors in the tumour microenvironment. Nat Rev Cancer 8: 929-941, 2008.

11. Klein T and Bischoff R: Active metalloproteases of the A disintegrin and metalloprotease (ADAM) family: Biological function and structure. J Proteome Res 10: 17-33, 2011. 
12. Saftig P and Lichtenthaler SF: The alpha secretase ADAM10: A metalloprotease with multiple functions in the brain. Prog Neurobiol 135: 1-20, 2015.

13. Vincent B: Regulation of the $\alpha$-secretase ADAM10 at transcriptional, translational and post-translational levels. Brain Res Bull 126: 154-169, 2016.

14. Janes PW,Saha N,Barton WA, Kolev MV,Wimmer-Kleikamp SH, Nievergall E, Blobel CP, Himanen JP, Lackmann M and Nikolov DB: Adam meets Eph: An ADAM substrate recognition module acts as a molecular switch for ephrin cleavage in trans. Cell 123: 291-304, 2005.

15. Reiss K, Maretzky T, Ludwig A, Tousseyn T, de Strooper B, Hartmann D and Saftig P: ADAM10 cleavage of N-cadherin and regulation of cell-cell adhesion and beta-catenin nuclear signalling. EMBO J 24: 742-752, 2005.

16. Maretzky T, Reiss K, Ludwig A, Buchholz J, Scholz F, Proksch E, de Strooper B, Hartmann D and Saftig P: ADAM10 mediates E-cadherin shedding and regulates epithelial cell-cell adhesion, migration, and beta-catenin translocation. Proc Natl Acad Sci USA 102: 9182-9187, 2005

17. Moss ML, Stoeck A, Yan W and Dempsey PJ: ADAM10 as a target for anti-cancer therapy. Curr Pharm Biotechnol 9: 2-8, 2008.

18. Zhang W, Liu S, Liu K, Wang Y, Ji B, Zhang X and Liu Y: A disintegrin and metalloprotease (ADAM) 10 is highly expressed in hepatocellular carcinoma and is associated with tumour progression. J Int Med Res 42: 611-618, 2014.

19. You B, Shan Y, Shi S, Li X and You Y: Effects of ADAM10 upregulation on progression, migration, and prognosis of nasopharyngeal carcinoma. Cancer Sci 106: 1506-1514, 2015

20. Guo J, He L, Yuan P, Wang P, Lu Y, Tong F, Wang Y, Yin Y, Tian J and Sun J: ADAM10 overexpression in human non-small cell lung cancer correlates with cell migration and invasion through the activation of the Notch1 signaling pathway. Oncol Rep 28: 1709-1718, 2012.

21. Wang YY, Ye ZY, Li L, Zhao ZS, Shao QS and Tao HQ: ADAM 10 is associated with gastric cancer progression and prognosis of patients. J Surg Oncol 103: 116-123, 2011.

22. Fu L, Liu N, Han Y, Xie C, Li Q and Wang E: ADAM10 regulates proliferation, invasion, and chemoresistance of bladder cancer cells. Tumour Biol 35: 9263-9268, 2014.

23. Jing P, Sa N and Xu W: miR-140-5p affects the migration and invasion of hypopharyngeal carcinoma cells by downregulating ADAM10 expression. Zhonghua Er Bi Yan Hou Tou Jing Wai Ke Za Zhi 51: 189-196, 2016 (In Chinese).
24. Siney EJ, Holden A, Casselden E, Bulstrode H, Thomas GJ and Willaime-Morawek S: Metalloproteinases ADAM10 and ADAM17 mediate migration and differentiation in glioblastoma sphere-forming cells. Mol Neurobiol: Aug 19, 2016 (Epub ahead of print).

25. Zepeda-Nuño JS, Guerrero-Velázquez C, Del Toro-Arreola S, Vega-Magaña N, Ángeles-Sánchez J, Haramati J, Pereira-Suárez AL and Bueno-Topete MR: Expression of ADAM10, Fas, FasL and soluble FasL in patients with oral squamous cell carcinoma (OSCC) and their association with clinical-pathological parameters. Pathol Oncol Res 23: 345-353, 2017.

26. Detre S, Saclani Jotti G and Dowsett M: A ‘quickscore' method for immunohistochemical semiquantitation: Validation for oestrogen receptor in breast carcinomas. J Clin Pathol 48: 876-878, 1995.

27. Graham JK, Kunze E and Hammerstedt RH: Analysis of sperm cell viability, acrosomal integrity, and mitochondrial function using flow cytometry. Biol Reprod 43: 55-64, 1990.

28. Chaffer CL and Weinberg RA: A perspective on cancer cell metastasis. Science 331: 1559-1564, 2011.

29. Acloque H, Adams MS, Fishwick K, Bronner-Fraser M and Nieto MA: Epithelial-mesenchymal transitions: The importance of changing cell state in development and disease. J Clin Invest 119: 1438-1449, 2009.

30. Kalluri R: EMT: When epithelial cells decide to become mesenchymal-like cells. J Clin Invest 119: 1417-1419, 2009.

31. Edwards DR, Handsley MM and Pennington CJ: The ADAM metalloproteinases. Mol Aspects Med 29: 258-289, 2008.

32. Reiss K and Saftig P: The 'a disintegrin and metalloprotease' (ADAM) family of sheddases: Physiological and cellular functions. Semin Cell Dev Biol 20: 126-137, 2009.

33. Weber S and Saftig P: Ectodomain shedding and ADAMs in development. Development 139: 3693-3709, 2012.

34. Sahin U, Weskamp G, Kelly K, Zhou HM, Higashiyama S, Peschon J, Hartmann D, Saftig P and Blobel CP: Distinct roles for ADAM10 and ADAM17 in ectodomain shedding of six EGFR ligands. J Cell Biol 164: 769-779, 2004.

35. van Tetering G, van Diest P, Verlaan I, van der Wall E, Kopan R and Vooijs M: Metalloprotease ADAM10 is required for Notch1 site 2 cleavage. J Biol Chem 284: 31018-31027, 2009.

36. Liu S, Zhang W, Liu K, Ji B and Wang G: Silencing ADAM10 inhibits the in vitro and in vivo growth of hepatocellular carcinoma cancer cells. Mol Med Rep 11: 597-602, 2015. 